\title{
Contribuições do capital social, laços fracos e redes sociais para a sustentabilidade: uma revisão sistemática com análise bibliométrica
}

\author{
Contributions of social capital, weak ties and social networks to sustainability: a systematic review
}

with bibliometric analysis

Contribuciones del capital social, lazos débiles y las redes sociales a la sostenibilidad: una revisión sistemática con análisis bibliométrico

Recebido: 01/01/2022 | Revisado: 06/01/2022 |Aceito: 12/01/2022| Publicado: 14/01/2022

Caren Stela Máximo Batista

ORCID: https://orcid.org/0000-0002-8664-0871

Universidade Federal de Rondônia, Brasil

E-mail: caren.batista@ifro.edu.br

Haroldo de Sá Medeiros

ORCID: https://orcid.org/0000-0002-8410-9913 Universidade Federal de Rondônia, Brasil

E-mail: Haroldo.medeiros@unir.br

Theophilo Alves de Souza Filho

ORCID: https://orcid.org/0000-0003-4331-0802

Universidade Federal de Rondônia, Brasil

E-mail: theophilo@unir.br

Mariluce Paes-de-Souza

ORCID: https://orcid.org/0000-0002-4202-0769

Universidade Federal de Rondônia, Brasil

E-mail: mariluce@unir.br

\begin{abstract}
Resumo
Com o desenvolvimento das ciências sociais emerge na literatura, a partir de 1980, pesquisas sobre o capital social presente nas relações dos indivíduos que constituem as redes sociais. Nesse cenário, ganha destaque a força dos laços (fracos e fortes) como elementos que contribuem para o sucesso das redes. A relevância da pesquisa consiste na necessidade de compreender qual o perfil da literatura sobre as contribuições dos temas capital social, laços fracos e redes sociais para a sustentabilidade. Assim, mostra-se relevante analisar as publicações que estudam a relação do capital social, redes sociais e laços fracos, com a sustentabilidade. A metodologia utilizada foi revisão sistemática da literatura com análise bibliométrica. O estudo teve como fonte as bases de dados Web of Science e Scopus. O estudo foi feito a partir da busca dos seguintes termos: "sustainability", "capital social", "laços fracos", "redes sociais", "social capital", "weak ties" e "social networks", o que gerou um total de 723 artigos, dos quais foi selecionada uma amostra de 531 para análise. Os resultados deste trabalho apontam para a relação existente entre a sustentabilidade e os temas capital social, laços fracos e redes sociais, suas características e seu estado da arte.
\end{abstract}

Palavras-chave: Sustentabilidade; Capital social; Laços fracos; Redes sociais.

\begin{abstract}
With the development of social sciences, from 1980 onwards, research on the social capital present in the relationships of individuals that constitute social networks emerged in the literature. In this scenario, the strength of ties (weak and strong) is highlighted as elements that contribute to the success of networks. The relevance of the research is the need to understand the profile of the literature on the contributions of the themes social capital, weak ties and social networks for sustainability. Thus, it is relevant to analyze publications that study the relationship of social capital, social networks and weak ties with sustainability. The methodology used was a systematic literature review with bibliometric analysis. The study was sourced from the Web of Science and Scopus databases. The study was based on the search for the following terms: "sustainability", "social capital", "weak ties", "social networks", "social capital", "weak ties" and "social networks", which generated a a total of 723 articles, from which a sample of 531 was selected for analysis. The results of this work point to the relationship between sustainability and the themes of social capital, weak ties and social networks, their characteristics and their state of the art.
\end{abstract}

Keywords: Sustainability; Social capital; Weak ties; Network.

\section{Resumen}

Con el desarrollo de las ciencias sociales, a partir de 1980, emergen en la literatura investigaciones sobre el capital social presente en las relaciones de los individuos que constituyen las redes sociales. En este escenario, se destaca la 
fuerza de los lazos (débiles y fuertes) como elementos que contribuyen al éxito de las redes. La relevancia de la investigación radica en la necesidad de comprender el perfil de la literatura sobre los aportes de los temas capital social, lazos débiles y redes sociales para la sostenibilidad. Por ello, es relevante analizar las publicaciones que estudian la relación del capital social, las redes sociales y los lazos débiles con la sostenibilidad. La metodología utilizada fue una revisión sistemática de la literatura con análisis bibliométrico. El estudio se obtuvo de las bases de datos Web of Science y Scopus. El estudio se basó en la búsqueda de los siguientes términos: "sustentabilidad", "capital social", "vínculos débiles", "redes sociales", "capital social", "vínculos débiles" y "redes sociales", lo que generó una total de 723 artículos, de los cuales se seleccionó una muestra de 531 para su análisis. Los resultados de este trabajo apuntan a la relación entre la sostenibilidad y los temas de capital social, lazos débiles y redes sociales, sus características y su estado del arte.

Palabras clave: Sostenibilidad; Capital social; Lazos débiles; Redes sociales.

\section{Introdução}

Com o desenvolvimento das ciências sociais, a partir de 1980, ganham destaque pesquisas sobre as redes sociais e o capital social presente nas relações dos seus atores. A maioria dessas pesquisas trazem na sua base teórica estudos de Pierre Bourdieu (1980) que contribuíram para a sociologia com pesquisas sobre o consumo e a vida econômica. Para Bourdieu (1980), o capital social é um recurso em diferentes níveis: de controle social, de benefícios mediatizados pela família e por redes não-familiares. Coleman (1988), define o capital social como um conjunto de elementos de uma dada estrutura social, que possibilita que os atores alcancem seus objetivos.

As redes sociais são definidas em estudos anteriores (Granovetter, 1973; Poliany, 1985), como um conjunto de nós ou atores ligados por relações sociais ou laços de tipos específicos. Nesse contexto, ganha destaque a teoria da força dos laços fracos, desenvolvida pelo sociólogo Granovetter em 1973, quando estudava a estrutura das redes sociais. O estudo de Granovetter concluiu que embora os laços fortes contribuam para um fluxo mais intenso na comunicação dentro da rede, os laços fracos não podem ser desprezados pois levam inovação à rede através de pontes entre os atores; os laços fracos podem por exemplo contribuir na aquisição de um novo emprego.

Relacionar a sustentabilidade com os temas acima apresentados é necessário pois as relações sociais estão presente em todas as ações que envolvem sustentabilidade, seja no planejamento ou desenvolvimento. Nesse sentido, algumas pesquisas começam a surgir, por exemplo o estudo de Kumar e Tiwary (2020) que aplicando as teorias de coprodução de conhecimento e de capital social, exploram o papel das empresas sociais na abordagem do desafio da pobreza energética, uma das muitas vertentes da sustentabilidade.

Apesar da importância cada vez maior da sustentabilidade nas ações de pequenas e grandes empresas, nas atividades que envolvem cadeias de suprimentos, no desenvolvimento de políticas públicas, nas práticas do cotidiano das pessoas, entre outros, não há muitas pesquisas que apresentem uma relação entre a sustentabilidade e os temas capital social, laços fracos e redes sociais. Nesse sentido, não é possível atualmente compreender por exemplo, como os laços fracos de uma rede social podem contribuir com a sustentabilidade e qual a relação do capital social nesse contexto. A fim de contribuir com os estudos acadêmicos que abordam as relações sociais e a sustentabilidade pareceu importante prospectar: Qual o panorama atual da literatura sobre as contribuições do capital social, laços fracos e redes sociais para a sustentabilidade? Destaca-se que o tema laços fracos foi escolhido devido à sua importante contribuição dentro das redes sociais, já comprovada na teoria de Granovetter (1973); e pareceu importante compreendê-lo no contexto da sustentabilidade.

A pesquisa tem como objetivo apresentar um panorama da literatura sobre as contribuições do capital social, laços fracos e redes sociais, para a sustentabilidade através de uma análise bibliométrica. Em termos de relevância teórica, este tudo traz ordem e clareza para o campo acadêmico, identificando o perfil da literatura e o padrão evolutivo do debate sobre o tema, auxiliando trabalhos acadêmicos semelhantes. Também contribui com a geração de um entendimento teórico que pode ser aproveitado por gestores nos processos de tomada de decisão. Ademais, incentiva o desenvolvimento de novas pesquisas com 
foco em cenários não identificados na literatura.

O artigo está organizado em 5 seções, incluindo esta breve introdução. A seção 2 apresenta os métodos utilizados para a realização da pesquisa. A seção 3 apresenta as análises e discussão dos resultados e na seção 4 as considerações finais da pesquisa.

\section{Metodologia}

A abordagem de pesquisa foi uma revisão sistemática da literatura que, segundo Galvão e Ricarte (2020), é uma modalidade de pesquisa, que segue protocolos específicos, buscando uma compreensão e dar alguma lógica a um grande corpus documental, especialmente, verificando o que funciona e o que não funciona num dado contexto. Adicionalmente a pesquisa sistemática precisa seguir uma metodologia explícita e ser possível de reproduzir. A análise dos dados foi conduzida a partir da análise bibliométrica.

Segundo Palludeto e Felipini (2019), a vantagem da análise bibliométrica é, sobretudo, a de permitir a construção de um mapa relativamente amplo das publicações, sob vários ângulos (critérios), processo que dificilmente poderia ser feito valendo-se de uma pesquisa bibliográfica tradicional. Ademais, a partir da análise bibliométrica, é possível também identificar principais linhas e tendências futuras no interior da literatura, mitigando os possíveis vieses e limites, inclusive de natureza física, de leitura e reflexão do próprio pesquisador que procura sistematizar o tema (Palludeto \& Felipini, 2019).

Esta pesquisa teve como foco o panorama da literatura de pesquisas que abordem os termos capital social, laços fracos, redes sociais e sustentabilidade e para atender este objetivo os procedimentos metodológicos foram realizados através das seguintes etapas: identificação dos trabalhos relacionados ao tema; seleção e exclusão conforme critérios pré-definidos; elegibilidade conforme aderência ao objeto de pesquisa.

A etapa de identificação foi realizada no dia 08 de julho de 2021, através de buscas nas bases de dados "Web of Science" e "Scopus", pois estas bases padronizam as referências bibliográficas e demais informações das publicações indexadas (fonte e tipos de documento, filiações autorais, detalhes de financiamento, entre outros) de forma a permitir a aplicação de técnicas bibliométricas com maior precisão (Palludeto \& Felipini, 2019). Inicialmente a busca foi feita usando os temos na língua portuguesa "laços fracos" and "capital social" and "redes sociais" and "sustentabilidade" simultaneamente, não sendo encontrado nenhum trabalho. Uma nova busca foi realizada utilizando os termos na língua inglesa "weak ties" and "social capital" and "social networks" and "sustainability", tendo como resultado 6 trabalhos. Optou-se então por excluir o termo "sustainability" da busca, tendo como resultado 723 trabalhos, dos quais foram eliminados 192 duplicados, resultando na amostra de 531 trabalhos, conforme Tabela 1. 
Tabela 1. Etapas de identificação, seleção e elegibilidade de artigos.

\begin{tabular}{|c|c|c|c|}
\hline Base de Dados & Termos & Critério & Artigos Identificados \\
\hline \multirow{3}{*}{ Web of Science } & $\begin{array}{l}\text { "laços fracos" and "capital } \\
\text { social" and "redes sociais" }\end{array}$ & Topic* & 0 \\
\hline & & & \\
\hline & $\begin{array}{l}\text { "weak ties" and "capital } \\
\text { social" and "social networks" }\end{array}$ & Topic & 437 \\
\hline \multirow{3}{*}{ Scopus } & $\begin{array}{l}\text { "laços fracos" and "capital } \\
\text { social" and "redes sociais" }\end{array}$ & $\begin{array}{c}\text { Article title, Abstract, } \\
\text { Keywords }\end{array}$ & 0 \\
\hline & & & \\
\hline & $\begin{array}{c}\text { "weak ties" and "capital } \\
\text { social" and "social networks" }\end{array}$ & $\begin{array}{c}\text { Article title, Abstract, } \\
\text { Keywords }\end{array}$ & 286 \\
\hline \multicolumn{2}{|c|}{ Total de artigos identificados } & & 723 \\
\hline \multicolumn{2}{|c|}{ Artigos duplicados } & & 192 \\
\hline \multicolumn{2}{|c|}{ Amostra final } & & 531 \\
\hline
\end{tabular}

Obs.: *Palavras incluídas title, abstract, author and keywords. Fonte: Elaborado pelos autores (2021).

Apesar da exclusão do termo "sustainability" a amostra final foi analisada com vistas a identificar os trabalhos que apresentavam pesquisas sobre capital social, laços fracos e redes sociais relacionados à sustentabilidade. Para análise dos dados, apresentados na próxima seção, foram utilizados os softwares Excel para apresentação dos dados em tabela, Word para a construção de gráficos e VOSviwer para agrupamento dos dados em clusters. O software VOSviwer dá ênfase na análise e na visualização de conjuntos grandes de dados bibliográficos a partir de uma abordagem distance-based (Palludeto \& Felipini, 2019). De acordo com van Eck e Waltman (2010), pequenas redes bibliográficas podem ser, em geral, representadas graficamente por programas como o Pajek (graph-based). Já redes maiores passariam a requerer um tratamento visual mais cuidadoso e aprofundado, exigindo um método de análise e visualização que fosse capaz de melhor extrair as informações da base sob observação, tal como o distance-based approach.

De modo amplo, os nodos da rede bibliográfica são postos de maneira que a distância entre eles indica aproximadamente a sua relação segundo determinados critérios de agregação, conformando um mapa. Segundo Palludeto e Felipini (2019), cada critério destaca um aspecto da rede formada pela bibliografia sobre o tema; esta pesquisa utilizou o critério de co-ocorrência que procura identificar elementos comuns aos textos da base de dados; como demonstrado mais à frente, ele será utilizado para identificar termos significativos, de modo que se possa sugerir subáreas dentro da base (Palludeto \& Felipini, 2019).

\section{Resultados e Discussão}

Os artigos analisados possuem data de publicação de 1994 a 2021, conforme Figura 1. De acordo com os dados, nos primeiros 10 anos a média de publicação anual era de 3 trabalhos. Nos anos de 2004 a 2014 a média anual subiu para 26,8 publicações, permanecendo em crescimento de 2015 a 2021 com uma média de 35 publicações anual, tendo como destaque o ano 2018 com 50 publicações. 
Figura 1. Distribuição anual da literatura.

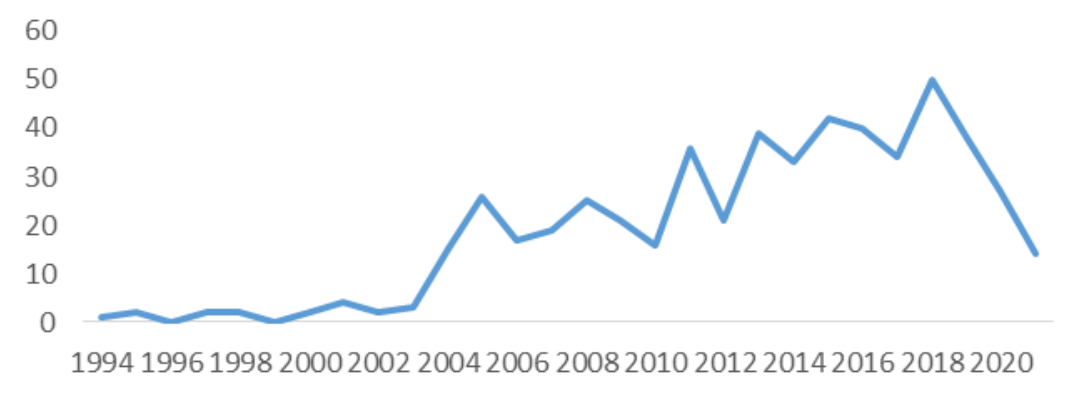

Fonte: Elaborado pelos autores (2021).

As publicações analisadas são de 51 países, mas a pesquisa optou por dar destaque aos 10 países com o maior número de trabalhos, sendo os três primeiros com origem nos países Estados Unidos (162), Inglaterra (43) e China (39), com proporções de 37\%, 9,8\%, e 8,9\% respectivamente, conforme Tabela 2.

Tabela 2. Os 10 países mais produtivos na pesquisa.

\begin{tabular}{cccc}
\hline Classificação & País & Publicações & $\%$ \\
\hline 1 & USA & 162 & 37.071 \\
2 & ENGLAND & 43 & 9.840 \\
3 & PEOPLES R CHINA & 39 & 8.924 \\
4 & GERMANY & 27 & 6.178 \\
5 & CANADA & 26 & 5.950 \\
6 & SPAIN & 26 & 5.950 \\
7 & AUSTRALIA & 20 & 4.577 \\
8 & NETHERLANDS & 20 & 4.577 \\
9 & SOUTH KOREA & 19 & 4.348 \\
10 & FRANCE & 14 & 3.204 \\
\hline
\end{tabular}

Fonte: Elaborado pelos autores (2021).

Nos Estados Unidos as três publicações de maior relevância científica incluem a revisão e análise do paradigma de rede emergente na pesquisa organizacional (Borgatti \& Foster, 2003); análise de estruturas de governança redundantes (Rowley et al., 2000), onde os autores concluem que laços fortes em uma rede de alianças estratégicas altamente interconectada impactam negativamente no desempenho da empresa; e a pesquisa de Ellison et al. (2011) que analisa as estratégias de conexão dos usuários do Facebook com o capital social.

$\mathrm{Na}$ Inglaterra as pesquisas mais relevantes envolvem temas como a dinâmica entre pobreza e exclusão; vizinhança e saúde e bem-estar considerando o papel das redes sociais e do capital social nos processos sociais envolvidos (Cattell, 2001); redes sociais de migrantes e laços fracos (Ryan, 2011). Através de um estudo de imigrantes poloneses em Londres Ryan (2011) argumenta que é necessária mais atenção às formas como os migrantes acessam, mantêm e constroem diferentes tipos de redes, em locais sociais variados, com pessoas diversas. Atenção insuficiente tem sido dada aos recursos reais que fluem entre esses laços ou aos tipos de relacionamento que se desenvolvem entre os atores envolvidos.

$\mathrm{Na}$ China o principal estudo que relaciona os temas capital social, laços fracos e redes sociais, é de Stam et al. (2014), que através de uma meta-análise, analisa o capital social de empreendedores e o desempenho de pequenas empresas. A pesquisa de Stam et al. (2014), através da análise de 61 amostras independentes, indica que a relação capital socialdesempenho foi positiva e significativa. Os tamanhos de efeito dos laços fracos foram menores do que os dos furos estruturais, enquanto a diversidade da rede teve o maior efeito positivo no desempenho (Stam et al., 2014). Os resultados apontaram que a 
relação capital social - desempenho depende da idade das pequenas empresas, da indústria e dos contextos institucionais em que operam e da rede específica ou das medidas de desempenho utilizadas (Stam et al., 2014).

Foram encontrados 171 artigos na área de Ciências Sociais, 89 artigos na área de Negócios, Gestão e Contabilidade, 38 artigos na área de Economia e Finanças, englobando estudos sobre estratégia, finanças, governança, entre outros, o que contribui para a discussão desses tipos de organização. Outras duas áreas de destaque são a Ciência da Computação e Sociologia e Psicologia, com discussão social de redes e comunidades e o papel do capital social e da teoria da força dos laços fracos nas mídias sociais.

Dos 89 periódicos relacionados à amostra da pesquisa, foram selecionados os dez com maior número de publicação, conforme Tabela 3. Não há periódico específico que trate do assunto, porém os periódicos de maior destaque são Elsevier (73), Sage (63) e Taylor e Francis (60), correspondendo a 16\%, 14\% e 13\% respectivamente, exposto na Tabela 3.

Tabela 3. Veículo de publicação dos artigos analisados.

\begin{tabular}{lcc}
\hline \multicolumn{1}{c}{ Periódico } & Quantidade & \% \\
\hline Elsevier & 73 & 16.705 \\
Sage & 63 & 14.416 \\
Taylor \& Francis & 60 & 13.730 \\
Wiley & 48 & 10.984 \\
Springer Nature & 31 & 7.094 \\
Emerald Group Publishing & 28 & 6.407 \\
Oxford Univ Press & 11 & 2.517 \\
IEEE & 9 & 2.059 \\
Cambridge Univ Press & 6 & 1.373 \\
Mdpi & 6 & 1.373 \\
\hline
\end{tabular}

Fonte: Elaborado pelos autores (2021).

Os temas capital social, laços fracos e redes sociais, formam um campo de pesquisa que abrange várias disciplinas e inclui pesquisadores de áreas como ciências sociais, gestão, economia, ciências da computação, sociologia e psicologia, artes e humanidades, engenharia e medicina. Todos os artigos identificados nas duas bases de dados online foram usados para a análise de acoplamento bibliográfico, através do VOS Viwer, onde foram gerados clusters que mostram a colaboração dos autores. O critério de acoplamento bibliográfico procura aproximar unidades que compartilham referências em comum. Na figura 2, cada nó representa um autor, o tamanho do nó representa o número de seus documentos, e a cor do nó representa o grupo ao qual o autor pertence com base no método de armazenamento em cluster padrão. As linhas na rede representam parcerias de contribuintes. Quanto mais larga for a linha, mais forte será a cooperação. 
Figura 2. Rede de co-autorias sobre capital social, laços fracos e redes sociais.

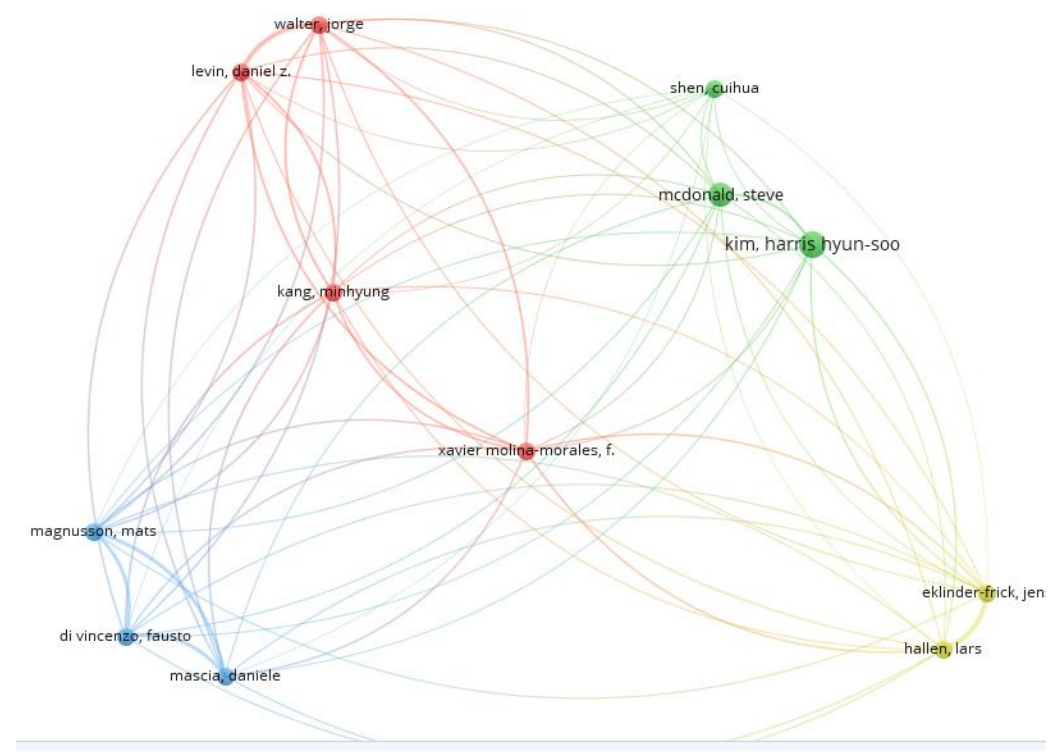

Fonte: Elaborado pelos autores (2021).

Ao considerar como critério as co-citações, são tomados propriamente como unidade de análise os autores. Vale dizer que caso um autor (ou conjunto de autores) possua mais de um texto referenciado pela base, os mesmos são agrupados na composição do mapa, aproximando-o de um autor (ou grupo de autores) cujos textos, também agrupados, aparecem citados conjuntamente. A Figura 3 apresenta 79 nós e 3 clusters e cada cluster é constituído por vários autores seminais que foram citados nos 531 artigos analisados.

Figura 3. Rede de co-citações.

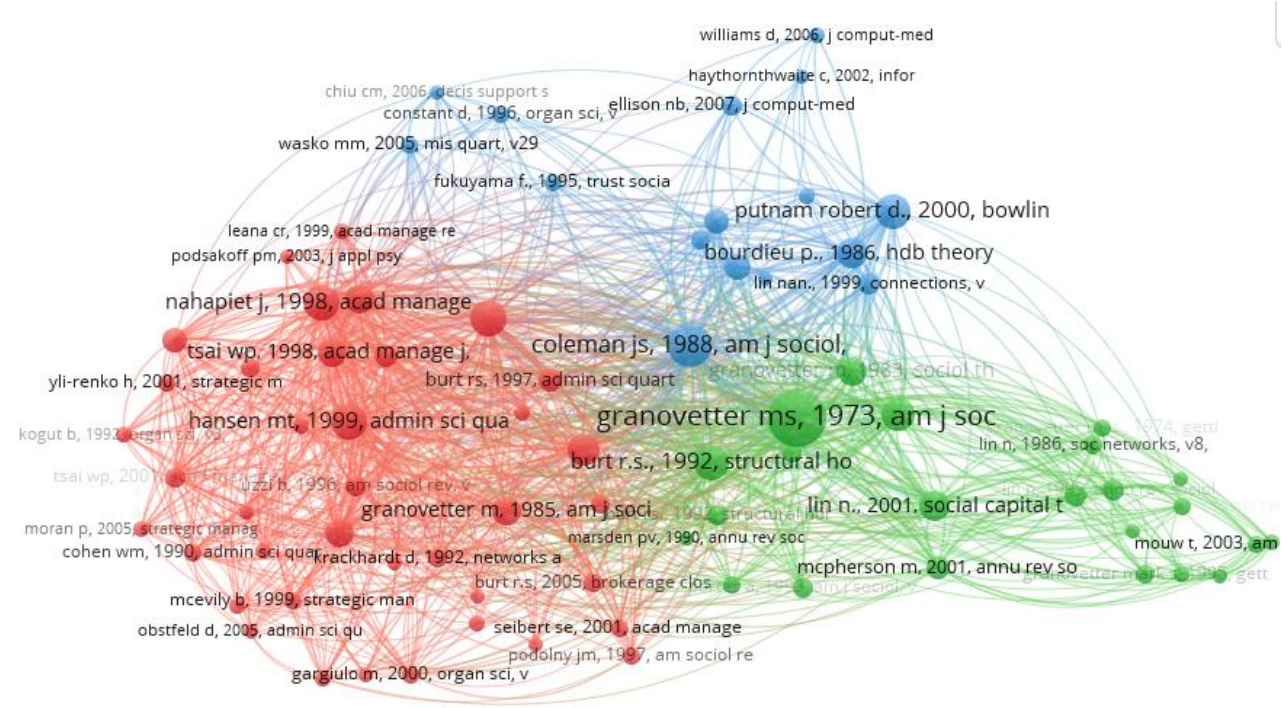

Fonte: Elaborado pelos autores (2021).

O cluster verde está representado por Granovetter (1973) com a obra “The strength of weak ties” aparece na tabela 6 com 306 citações. O cluster azul tem como principal autor seminal Coleman (1988) com a obra "Social Capital in the Creation of Human Capital" e possui 174 citações. O cluster vermelho é representado por Nahapiet (1998) com a obra "Social Capital, 
Intellectual Capital, and the Organizational Advantage" com 125 citações. Além do destaque dado aos autores mais citados, também foi possível, durante as análises, encontrar 59 autores citados de 20 a 51 vezes, o que demonstra que a discussão científica do tema abordado é ainda contínua e centralizada

Também atentou-se para os termos que as publicações compartilham entre si, relacionando ao ano de publicação. Com efeito, ao considerar as expressões que mais ocorrem na literatura e reuni-las, é possível não apenas complementar os agrupamentos já observados, como também obter uma aproximação relativamente fidedigna do conteúdo de cada agrupamento em termos de objeto de análise. Nesse critério, o objetivo, portanto, é identificar termos e expressões significativos nos resumos, palavras-chave e títulos das publicações da rede, o que pode ser visto na figura 4. Na busca, foram selecionados artigos com o mínimo de 5 palavras-chave, tendo como resultado 165 palavras-chave agrupadas em 5 clusters. A Figura 4 mostra tendências na co-ocorrência de palavras-chave dos trabalhos sobre capital social, laços fracos e redes sociais nos últimos anos; o resultado desta análise indica tendências promissoras que podem auxiliar futuros trabalhos sobre o tema.

Figura 4. Agrupamento por co-ocorrência de termos por ano de publicação.

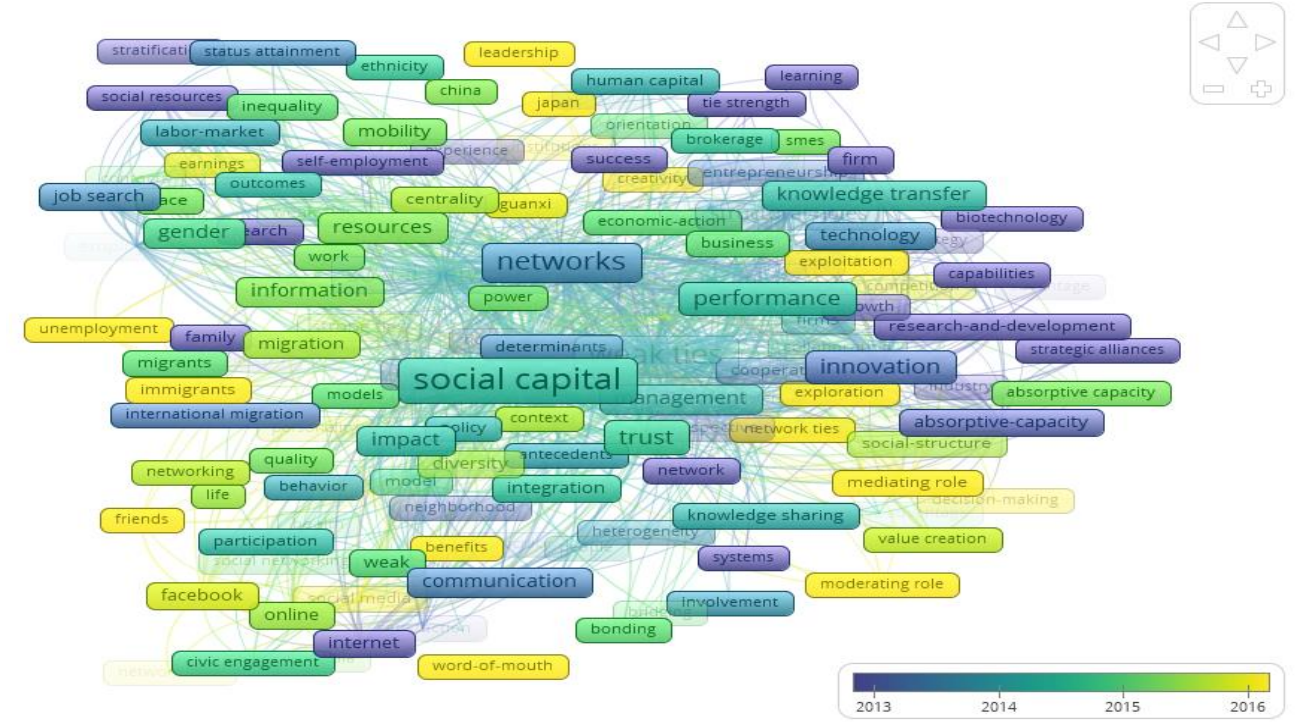

Fonte: Elaborado pelos autores (2021).

O gradiente de cor (azul para amarelo) na figura 4 representa tendências na co-ocorrência de palavras-chave de 2013 a 2016. De 2013 a 2014 é possível perceber que os estudo termos como: capital social, social resources, research and development, strategic aliances, networks, sistems, internet, tie strengh, learning, job research, entre outros, dando destaque à abordagem de Granovetter sobre a importância dos laços fracos entre os indivíduos na aquisição de um novo emprego. De 2014 a 2015 são percebidos termos como: impact, trust, participation, knowledge sharing, performance, labor-market, human capital, através dos quais infere-se uma linha de pesquisa voltada para as relações humanas com ênfase na troca de conhecimento entre os atores das redes sociais e na atuação no mercado de trabalho em geral. A partir de 2016 a tendência nas pesquisas volta-se para a importância dos laços como moderadores nas mídias sociais para disseminação da inovação, através do marketing boca a boca; são percebidos os seguintes termos: facebook, technology, weak-ties, mediating role, word of mouth. O termo rede social aparece na maioria destes trabalhos, referindo-se às relações presentes nas mídias sociais.

\section{Considerações Finais}

$\mathrm{O}$ presente artigo buscou fornecer um panorama relativamente abrangente da progressiva literatura acerca da integração da capital social, laços fracos e redes sociais. Para isso foi realizada uma revisão sistemática na literatura, com 
análise bibliométrica. O estudo foi realizado com uma amostra de 531 artigos de onde foi possível inferir que existe uma vasta literatura que associa os termos analisados. A maioria das pesquisas apresentou esta relação dando destaque à migração dos indivíduos no mercado de trabalho e abordagens com foco nas mídias sociais. Dessa forma, é possível inferir que existe uma lacuna na literatura sobre a integração desses temas com vistas a outros campos de pesquisa como desenvolvimento organizacional e desenvolvimento sustentável.

Nesse sentido, as recomendações para pesquisas futuras incluem estudos que mostrem a associação do capital social, redes sociais e força dos laços com foco no desenvolvimento organizacional. Sugere-se ainda que futuros estudos investiguem a contribuição do capital social, das redes sociais e a força dos laços para o desenvolvimento sustentável. Ainda, percebe-se a necessidade de estudos de caso que mostrem como efetivamente o capital social e a teoria da força dos laços são percebidas nas organizações.

Essa pesquisa contribuiu para o mapeamento da literatura sobre a associação dos termos capital social, laços fracos e redes sociais que pode auxiliar trabalhos acadêmicos semelhantes, bem como com a geração de um entendimento teórico que pode ser aproveitado por gestores nos processos de tomada de decisão. Ademais, incentiva o desenvolvimento de novas pesquisas com foco em cenários não identificados na literatura.

\section{Referências}

Booth, A. (2006). "Brimful of STARLITE": toward standards for reporting literature searches. Journal of the Medical Library Association, 94(4), 421.

Bourdieu, P. (1980). Le capital social: notes provisoires. Actes de la recherche en sciences sociales, 31(1), 2-3.

Cattell, V. (2001). Poor people, poor places, and poor health: the mediating role of social networks and social capital. Social science \& medicine, 52(10), 1501-1516.

Coleman, J. S. (1994). Foundations of social theory. Harvard university press.

Coleman, J. S. (1988). Social capital in the creation of human capital. American journal of sociology, 94, S95-S120.

Creswell, J. W., \& Creswell, J. D. (2021). Projeto de pesquisa-: Métodos qualitativo, quantitativo e misto. Penso Editora.

Davidsson, P., \& Honig, B. (2003). The role of social and human capital among nascent entrepreneurs. Journal of business venturing, $18(3)$, $301-331$.

Ellison, N. B., Steinfield, C., \& Lampe, C. (2011). Connection strategies: Social capital implications of Facebook-enabled communication practices. New media \& society, 13(6), 873-892.

Foster, J. (2019). Thank you for being a friend: The roles of strong and weak social network ties in attracting backers to crowdfunded campaigns. Information Economics and Policy, 49, 100832.

Fukuyama, F. (1996). Confiança: as virtudes sociais e criação da prosperidade. Rocco.

Granovetter, M. S. (1973). The strength of weak ties. American journal of sociology, 78(6), 1360-1380.

Granovetter, M. (1985). Economic action and social structure: The problem of embeddedness. American journal of sociology, 91(3), 481-510.

Growiec, K., Growiec, J., \& Kamiński, B. (2018). Social network structure and the trade-off between social utility and economic performance. Social Networks, 55, 31-46.

Kaufman, D. (2012). A força dos “laços fracos” de Mark Granovetter no ambiente do ciberespaço. Galaxia, (23), 207-218.

Kim, T. T., Lee, G., Paek, S., \& Lee, S. (2013). Social capital, knowledge sharing and organizational performance: what structural relationship do they have in hotels?. International Journal of Contemporary Hospitality Management.

Kumar, P., \& Tiwary, N. (2020). Role of Social Enterprises in Addressing Energy Poverty: Making the Case for Refined Understanding through Theory of CoProduction of Knowledge and Theory of Social Capital. Sustainability, 12(20), 8533.

Lemos, E., Filho, T., Medeiros, H., \& Reis, S. (2021). Laços Fracos em Unidades de Conservação da Amazônia Brasileira: uma revisão sistemática. Revista de Administração e Negócios da Amazônia.

Li, Y., Pickles, A., \& Savage, M. (2005). Social capital and social trust in Britain. European sociological review, 21(2), 109-123.

Mertens, F., Távora, R., Fonseca, I. F. D., Grando, R., Castro, M., \& Demeda, K. (2011). Redes sociais, capital social e governança ambiental no Território Portal da Amazônia. Acta Amazônica, 41, 481-492. 
Research, Society and Development, v. 11, n. 1, e53211125284, 2022

(CC BY 4.0) | ISSN 2525-3409 | DOI: http://dx.doi.org/10.33448/rsd-v11i1.25284

Palludeto, A. W. A., \& Felipini, A. R. (2019). Panorama da literatura sobre a financeirização (1992-2017): uma abordagem bibliométrica. Economia e sociedade, 28, 313-337.

Portes, A. (2000, March). The two meanings of social capital. In Sociological fórum. 15(1), 1-12. Kluwer Academic Publishers-Plenum Publishers.

Putnam, R. D. (Ed.). (2004). Democracies in flux: The evolution of social capital in contemporary society. Oxford University Press, USA.

Rodrigues, S. B., \& Child, J. (2012). Building social capital for internationalization. Revista de Administração Contemporânea, 16, 23 -38.

Rowley, T., Behrens, D., \& Krackhardt, D. (2000). Redundant governance structures: An analysis of structural and relational embeddedness in the steel and semiconductor industries. Strategic management journal, 21(3), 369-386.

Ryan, L. (2011). Migrants' social networks and weak ties: accessing resources and constructing relationships post-migration. The Sociological Review, 59(4), $707-724$.

Shiau, W. L., Dwivedi, Y. K., \& Yang, H. S. (2017). Co-citation and cluster analyses of extant literature on social networks. International Journal of Information Management, 37(5), 390-399.

Stam, W., Arzlanian, S., \& Elfring, T. (2014). Social capital of entrepreneurs and small firm performance: A meta-analysis of contextual and methodological moderators. Journal of business venturing, 29(1), 152-173.

Thomas, A., \& Paul, J. (2019). Knowledge transfer and innovation through university-industry partnership: An integrated theoretical view. Knowledge Management Research \& Practice, 17(4), 436-448.

Zheng, J., Wu, G., \& Xie, H. (2017). Impacts of leadership on project-based organizational innovation performance: The mediator of knowledge sharing and moderator of social capital. Sustainability, 9(10), 1893. 